\title{
Computer Interface to Accurately Determine Fermi Energy and Fermi Temperature of Materials
}

\author{
Prashant Kumar Tripathi ${ }^{1}$ \\ Gangadharan K V² \\ ${ }^{1}$ Research Scholar \\ ${ }^{2}$ Professor \\ Department of Mechanical Engineering \\ National Institute of Technology Karnataka
}

\begin{abstract}
Determine Fermi energy and Fermi temperature of different materials by studying the resistance variation at different temperature is an important task to find and develop new semiconductors. Authors developed computer application with data acquisition system makes the task easy by system timed reading method and improve accuracy of the experiment. The major part of a virtual instrument is realized by software, which can be modified quickly and easily. As one of the most widespread and most efficient virtual instrumentation environment, LabVIEW was used to develop GUI of the experiment. This postgraduate level physics experiment was set as web published remote experiment to improve critical thinking and problem solving skills of students by engaging them through individual experimentation. The virtual experiment setup is validated by conducting set of experiment on different materials. The visibility of experiments becomes better, reliability is improved. The same setup can be used to determine Fermi energy and Fermi temperature of Nano materials where precise calculations are required and a virtual instrument plays an important role.
\end{abstract}

\section{General Terms}

Experimental Computer Application, Virtual Instrumentation.

\section{Keywords}

Remote laboratories;Fermi energy;Virtual instrumentation.

\section{INTRODUCTION}

Practical skills are of high importance in tertiary engineering and science education. During practical sessions students test and apply their theoretical knowledge in practical situations. They also develop hands-on skills essential for graduates to be successful in their future professional career. New technologies developed over the past two decades have enabled practical laboratories to be complemented and to some extent replaced with virtual and remote laboratories. A virtual instrument consists of a computer equipped with powerful application software, cost-effective hardware such as PC plug-in boards, and driver software, which together outperform the functions of traditional instruments for test and automation. Virtual instruments represent a fundamental shift from traditional hardware centric instrumentation systems to software centric systems that exploit the computing power, productivity, display and connectivity capabilities of modern day computers [1]. In this research virtual instrumentation concept was used for developing the real time experiments for semiconductors and other new materials to determine Fermi
Energy and Fermi Temperature by computer aid. The concept of the Fermi energy is a crucially important concept for the understanding of the electrical and thermal properties of solids. Both ordinary electrical and thermal processes involve energies of a small fraction of an electron volt. But the Fermi energies of metals are in the order of electron volts. This implies that the vast majority of the electrons cannot receive energy from those processes because there are no available energy states for them to go to within a fraction of an electron volt of their present energy. Limited to a tiny depth of energy, these interactions are limited .to "ripples on the Fermi Sea"[8]. Several researchers have investigated the use of computers, specifically simulation and visualization technology in experiments. Foley had shown that computer simulation and visualization can be very effective in enhancing student learning if the interface is based on education research [2].

Foley's study for engineering education improvement by computer application development and concluded that visualization tools facilitate understanding and retention of key concepts.

In addition, the continuum models allow us to study the electron states at large distances from the defects. The first attempts to analyze the electronic states of carbon structures with hyperboloid geometry within the continuum model were made in $[4,10]$. The main finding was the existence of the normalized electron state at the Fermi level. However, any information about electronic states near the Fermi energy was lacking.

Modern measurement systems for data acquisition and processing in research combine three basic functions:

\section{A. Data acquisition:}

This comprises a number of measured quantities, characterizing the behavior of the object of measurement; they are sampled simultaneously or sequentially, in most cases multiplexing the measured signals through several analogue channels $(8,16,32$ or more) for further conversion by a common analogue to digital converter. This function is implemented in hardware by DAQ-systems (Data Acquisition Systems).

B. Data analysis:

By means of algorithms for processing the results from multiple, aggregate or combined measurements with specific procedures for measurement and calculation which eliminate systematic errors, depending on the measured quantity and the environment within which it is monitored. Usually this function requires performing a large amount of computational and logical operations for reducing the initial indetermination 
of the quantity under measurement by comparing it with what is called best value and interval of residual indetermination standard deviation.

\section{Data presentation:}

Most often this comprises visual relations among measured data in graphical or tabular form. Their suitable visualization has a certain (often decisive) impact on the quality of the carried out measurement process. Usually when conducting scientific research one has to "experiment" with scaling of graphs, approximation of the processed signals and visualization of the functional relations.

\section{LabVIEW}

It is a graphical programming environment used by researchers and engineers to develop sophisticated measurement, test, and control systems using intuitive graphical icons and wires that resemble a flowchart. It provides integration of hardware and software for any real time applications.

For our experiments we have used LabVIEW 9.0, C-series module NI 9219 with K-type thermocouple, NI USB carrier 9162, Traditional Fermi Energy measurement Instrument, and Keithley 2400 Sourcemeter.

\section{INSTRUMENTATION AND SETUP}

The Fermi energy, of a substance is a simple concept with far reaching results. It is a result of the Pauli Exclusion Principle when the temperature of a material is lowered to absolute zero. By this principle, only one electron can inhabit a given energy state at a given time. When the temperature is lowered to absolute zero all of the electrons in the solid attempt to get into the lowest available energy level. As a result, they form a sea of energy states known as the Fermi Sea. The highest energy level of this sea is called the Fermi energy or Fermi level. At absolute zero no electrons have enough energy to occupy any energy levels above the Fermi level. In metals the Fermi level sits between the valence and conduction bands. The size of the so called band gap between the Fermi level and the conduction band determines if the metal is a conductor, insulator or semiconductor

Once the temperature of the material is raised above absolute zero the Fermi energy can be used to determine the probability of an electron having a particular energy level. As equation 1 shows, the probability that an energy state is filled by an electron depends on the Fermi energy.

$$
E_{f}=\frac{1.36 \times 10^{-15}}{1.6 \times 10^{-19}} \sqrt{\frac{\rho A S}{L}} \mathrm{eV}
$$

Where, $E_{f}$ - Fermi energy of copper $(\mathrm{eV})$

$$
\begin{aligned}
& \rho \text { - Density of copper }\left(\mathrm{kgm}^{-3}\right) \\
& \text { A - Area of cross section }\left(\mathrm{m}^{-2}\right) \\
& \mathrm{L} \text { - Length of copper wire }(\mathrm{m}) \\
& \mathrm{S} \text { - Slope of the curve plotted R versus T }
\end{aligned}
$$

In metals, the Fermi energy gives us information about the velocities of the electrons which participate in ordinary electrical conduction. The amount of energy which can be given to an electron in such conduction processes is on the order of micro electron volts, so only those electrons very close to the Fermi energy can participate. The Fermi velocity of these conduction electrons can be calculated from the Fermi energy. The Fermi energy also plays an important role in understanding the mystery of why electrons do not contribute significantly to the specific heat of solids at ordinary temperatures, while they are dominant contributors to thermal conductivity and electrical conductivity. Since only a tiny fraction of the electrons in a metal are within the thermal energy kT of the Fermi energy, they are "frozen out" of the heat capacity by the Pauli principle. At very low temperatures, the electron specific heat becomes significant.

The resistance measurements were carried out on a copper sample. The experimental set up consists of,

- Traditional ammeter and voltmeter instrument with heater.

- Keithley 2400 Sourcemeter.

- $\quad$ NI 9219 universal analog input module.

- $\quad$ NI USB carrier 9162.

- K-type thermocouple.

- Constant power supply for heater.

- $\quad$ PC with LabVIEW 9.0.

The block diagram of the experimental setup is shown in Figure 1. It consists of a four probe arrangement for resistivity measurement fitted with a heater having separate power supply. The maximum temperature achievable is $800 \mathrm{~K}$.

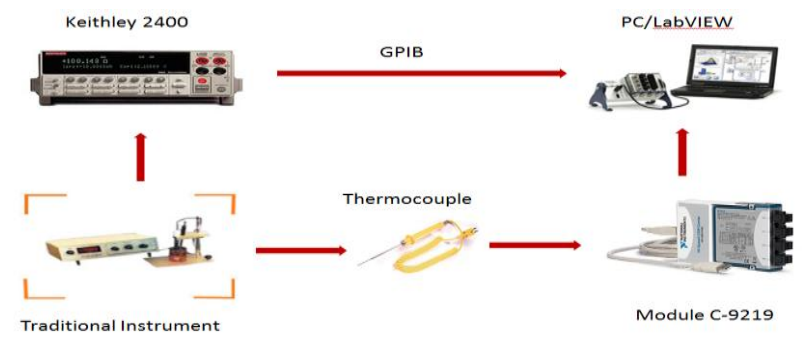

Figure 1: Block diagram of resistivity measurements.

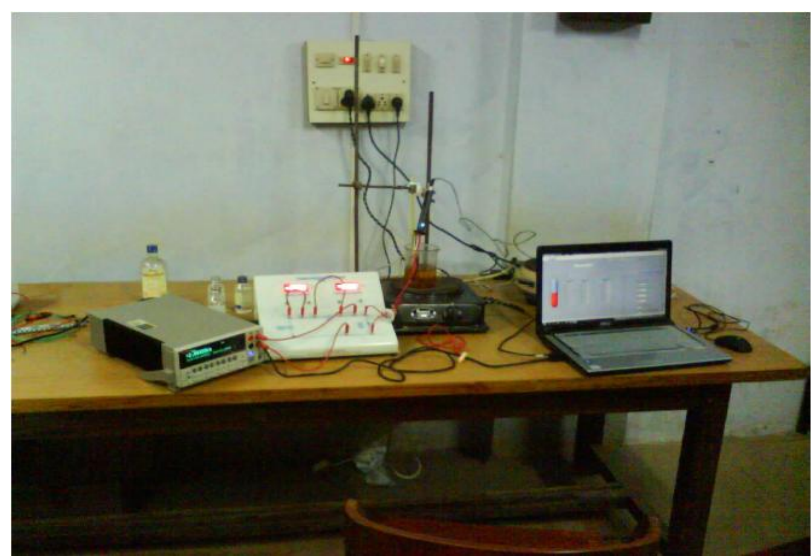

Figure 2: Experimental setup with computer Interface

The leads from the traditional Fermi setup was connected to the Keithley 2400 Sourcemeter. The Sourcemeter in turn connected to the computer via GPIB. The drivers used to operate the Sourcemeter remotely are obtained from National Instruments website. The drivers were modified according to our experiment to perform resistance measurement with change in temperature. A thermocouple (K-type) was kept in contact with the sample and the leads from it were given to NI 9219 module. The module was placed in NI 9162 USB carrier data acquisition system and connected to the computer via Universal serial bus. The experimental setup is shown in Figure 2. 


\section{COMPUTER PROGRAMMING AND VISA INTERFACE}

LabVIEW program (front panel and block diagram) for the experiment is shown in Figure $3 \& 4$. The program was developed according to our need using drivers from Keithley 2400. The left column in the front panel shows the VISA resource name and input parameters to be adjusted before any measurement. VISA resource name is to be set by the user according to the instrument connected to the particular data transfer cable. In the present experiment the data cable used was GPIB (KUSBA 488) and the VISA address was set to "GPIB11: 11: INSTR". The input parameters include Number of readings, delay time, sample count. Number of readings indicates the number of reading to be recorded, which can be set according to user's choice. Delay time is the time delay between two successive iterations. Sample count is the number of samples to be recorded in each run.

In source settings part user can set the source to either current or voltage and set the desired compliance limit. The output part displays the array of readings recorded for the latest iteration. " $i$ " displays the present iteration. On the right hand side in the output section there are three graphs which were displayed in a single multi tab window. The graphs are: (i) resistance graph versus time, (ii) temperature graph versus time and (iii) resistance versus temperature graph.

Where, $\mathrm{V}$ is voltage and $\mathrm{I}$ is the current. The readings recorded for all iterations were written on a LabVIEW measurement file. Temperature data acquired from thermocouple and resistance readings from the Keithley instrument were written in files on a user-defined location.

\section{RESULTS AND CONCLUSION}

The Fermi energy and Fermi temperature measurements were carried out from $500 \mathrm{~K}$ to room temperature. All the temperature readings from the thermocouple and current, voltage and resistance readings from the Keithley 2400 are recorded. Resistance values were plotted against temperature values. The sample used was copper wire $(3.6 \mathrm{~m}$ length and radius $0.26 \mathrm{~mm}$ ). The resistance value showed nearly constant value at room temperature measurements for a large number of readings. As the temperature decreased from higher temperature to the room temperature there was a decrease in the resistance of the sample as expected.

Figure 5 shows a snapshot recorded during the measurement. It shows a decreasing trend line for resistance of the sample. Slope of the graph is calculated by LabVIEW curve fitting method. Source settings are set to current source with $47 \mathrm{~mA}$ as the test current and $30 \mathrm{~V}$ as the compliance level. The resistance graph displays ten samples for all iteration. In the Figure 3 the combine results of all iteration are displayed and the graph shows ten values of the resistance measurements (Temperature along the $\mathrm{x}$-axis). The resistance measured is displayed along y-axis in the units of $\Omega$. From the slope of graph the Fermi Energy is calculated in back end and shown in numeric indicator.

Fermi Energy is an important concept in the study of metals, insulators, semiconductors, and white dwarf stars, and in understanding other material properties such as thermal conductivity. Knowledge of the Fermi energy of a material has allowed deeper study in many areas of science, such as the thermal and electrical properties of non-conductive materials, like diamonds, electron tunneling, and the kinetics of free electrons.

The concept of Fermi energy has allowed us to understand more about the interactions of electrons, and the correlation between energy states and physical properties.

An observation at the resistance values in the output section shows that the resistance has decreased and it keeps on decreasing with temperature. A number of measurements were performed on the sample to check the repeatability and it was observed that the resistance values and the change in resistances are almost same. Figure 6 shows a graph showing final measurements of temperature versus resistance which clearly displays the rise in resistance with temperature.

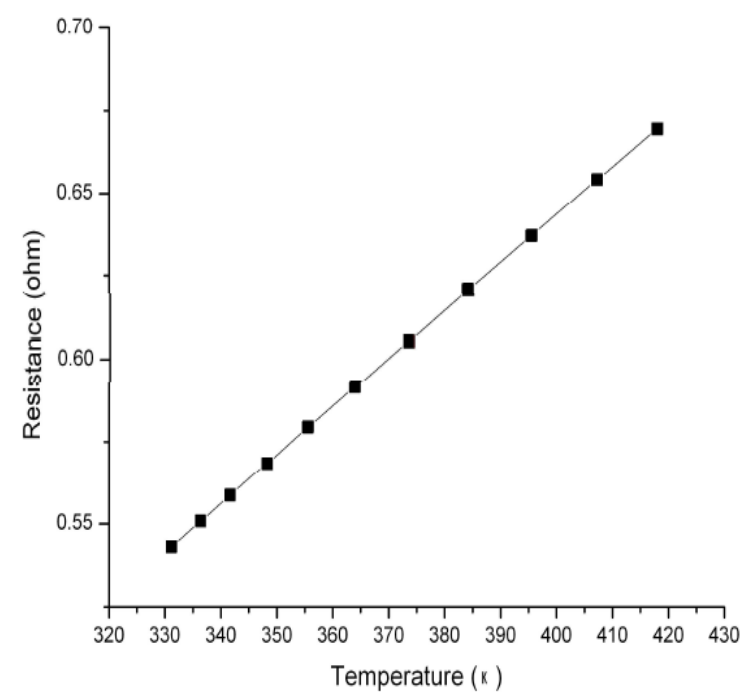

Figure 6: A Temperature vs. Resistance graph. 


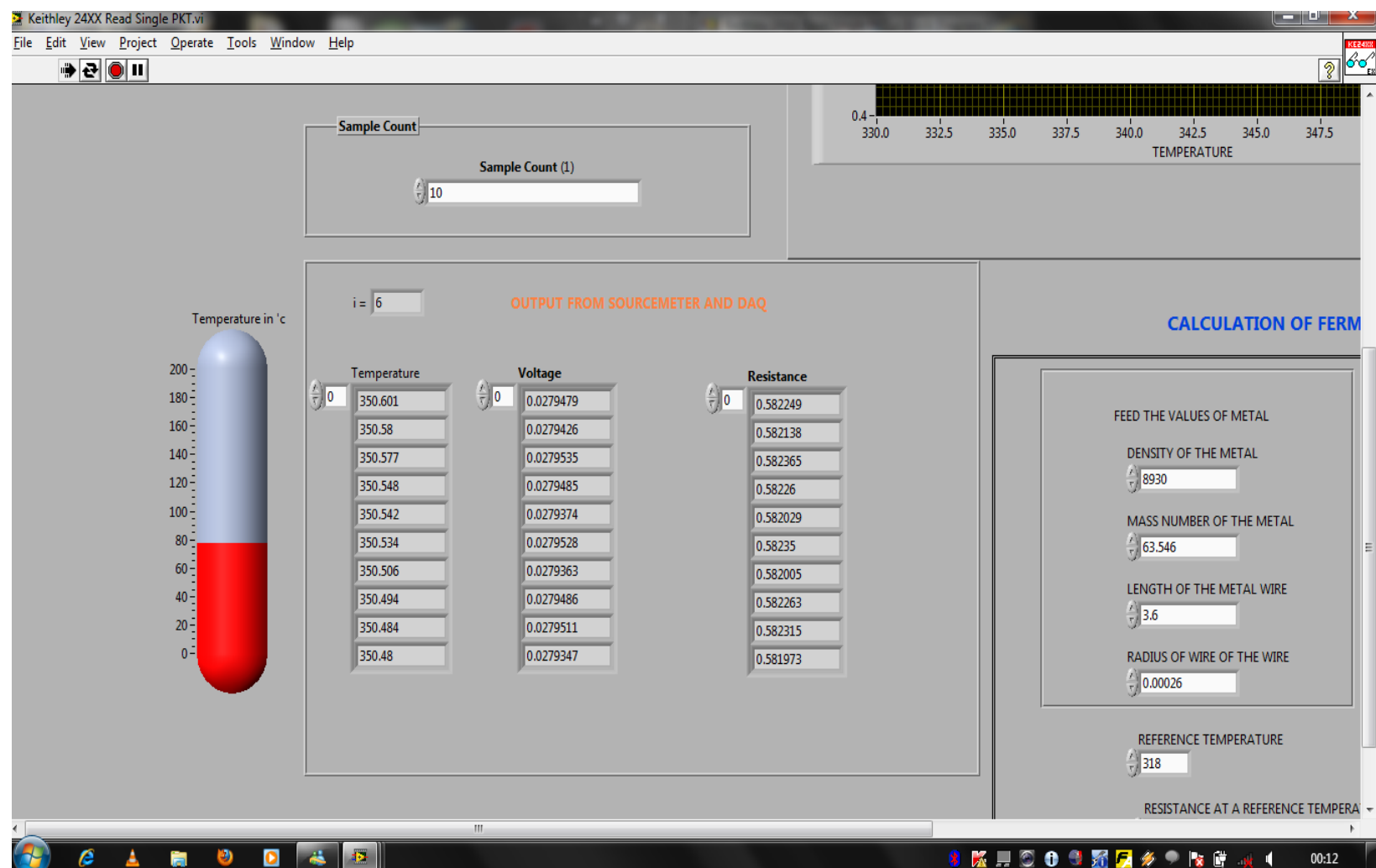

Figure 3: Front panel of Computer Application in the LabVIEW.

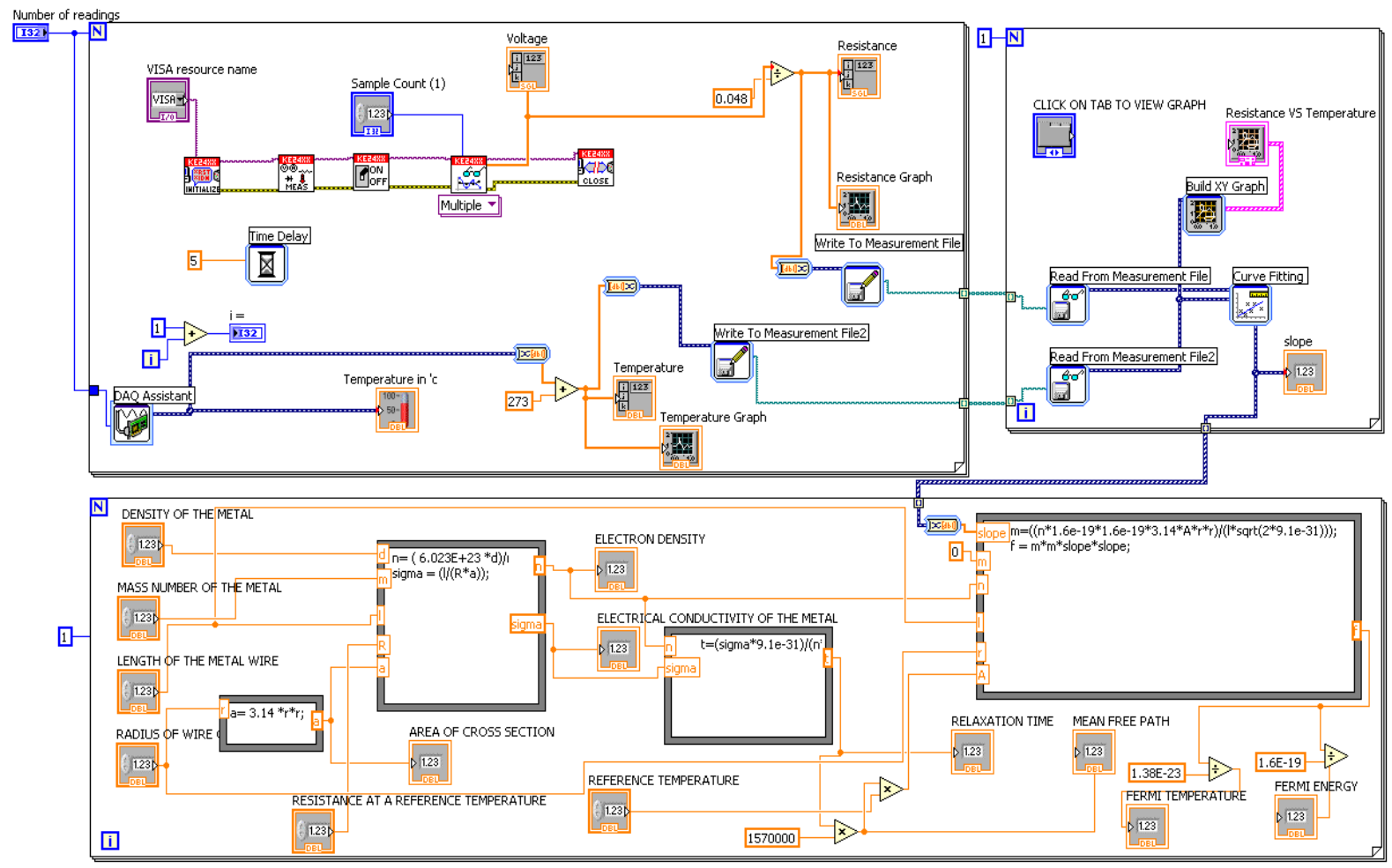

Figure 4: Block diagram with 'G'- programing in LabVIEW 


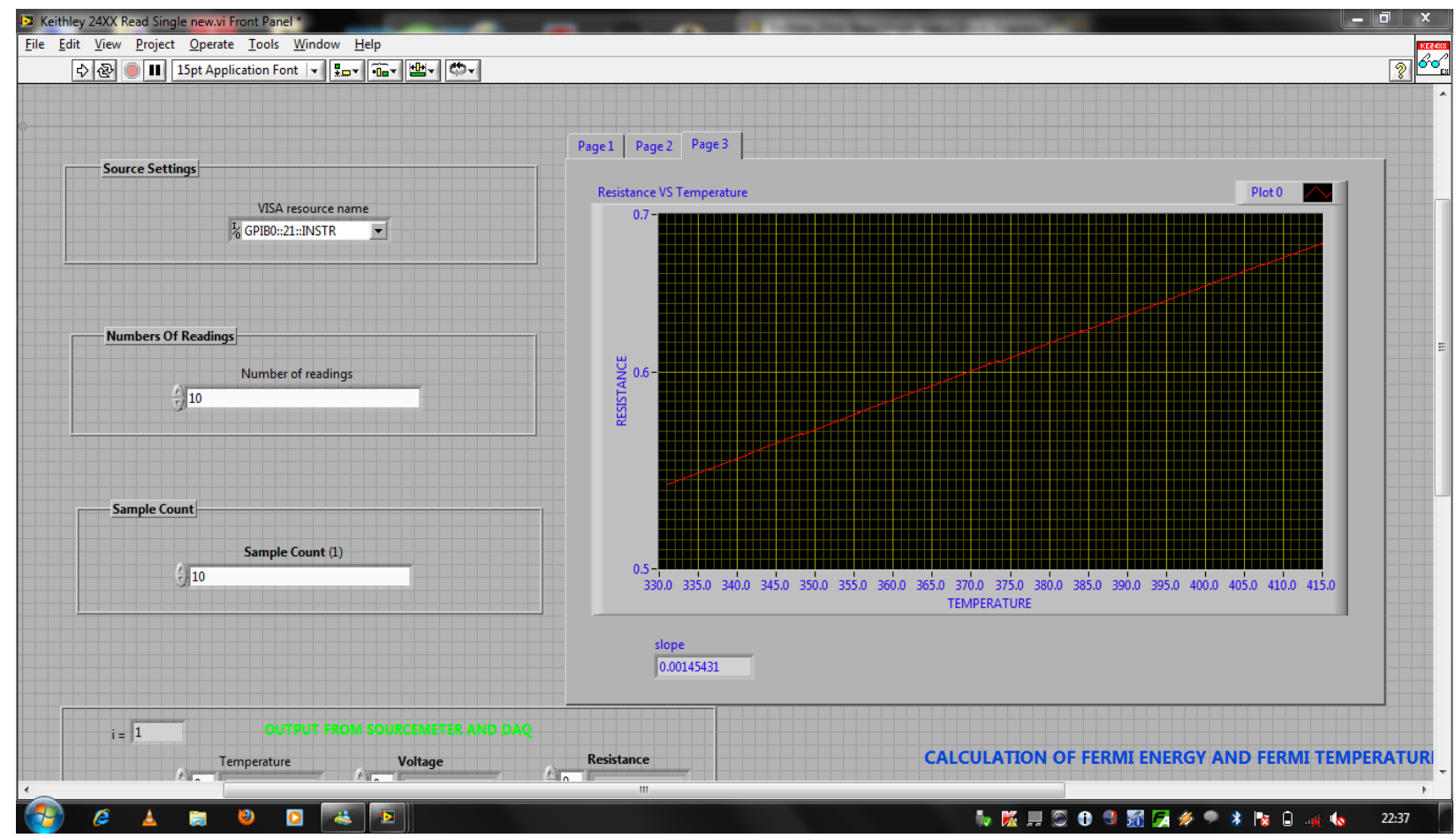

Figure 5: Front panel of computer application with graph of resistance vs. temperature.

Table 1: Compression of Literature data versus computer interface data

\begin{tabular}{|c|c|c|c|c|c|c|}
\hline \multirow{2}{*}{ Element } & \multicolumn{3}{|c|}{ From Literature } & \multicolumn{2}{c|}{ Computer Interfaced Experiment } \\
\cline { 2 - 7 } & $\begin{array}{c}\text { Fermi } \\
\text { Energy } \\
\mathrm{eV}\end{array}$ & $\begin{array}{c}\text { Fermi } \\
\text { Temperature } \\
\mathrm{X} 10^{4} \mathrm{~K}\end{array}$ & $\begin{array}{c}\text { Fermi } \\
\text { velocity } \\
\mathrm{X} 10^{6} \mathrm{~m} / \mathrm{s}\end{array}$ & $\begin{array}{c}\text { Fermi } \\
\text { Energy } \\
\mathrm{eV}\end{array}$ & $\begin{array}{c}\text { Fermi } \\
\text { Temperature } \\
\mathrm{X} 10^{4}\end{array}$ & $\begin{array}{c}\text { Fermi } \\
\text { Velocity } \\
\mathrm{X} 10^{6} \mathrm{~m} / \mathrm{s}\end{array}$ \\
\hline $\mathrm{Cu}$ & 7 & 8.1 & 1.57 & 6.81 & 8.165 & 1.552 \\
\hline $\mathrm{Sn}$ & 10.2 & 11.8 & 1.90 & 9.82 & 11.852 & 1.892 \\
\hline $\mathrm{Zn}$ & 9.47 & 11.0 & 1.83 & 9.44 & 11.251 & 1.825 \\
\hline $\mathrm{Al}$ & 11.7 & 13.6 & 2.03 & 11.65 & 13.523 & 2.125 \\
\hline
\end{tabular}

\section{CONCLUSION}

A computer application was developed for virtual instrumentation of Fermi Energy measurements. Temperature dependence of resistance was studied for a copper metal. Calculated Fermi Energy (6.8eV) for the taken metal was in well agreement with Literature ones $(7 \mathrm{eV})$. From the graph slope was calculated and used for Fermi Energy calculation. It was also found that the measured values are precise. The same setup can be used for Nano materials where precise calculations are required and a virtual instrument plays an important role.

We demonstrated the applicability of virtual instrumentation technology in classroom experiments. The traditional demonstration tools aged, broke down or disappeared. Now, many tools can be replaced by single hardware and the respective virtual instrument. The visibility of our experiments becomes better, reliability will be improved. Experiments data can be interrelated remotely through the internet.

\section{ACKNOWLEDGMENTS}

We sincerely thank Department of Mechanical Engineering NITK Surathkal in the implementation of online laboratories and the generous support provided by the Centre for System Design: A Centre of excellence at NITK Surathkal.

\section{REFERENCES}

[1] James, T. "The future of virtual instrumentation, virtual instrumentation Scientific Computing World": May / June 2004.

[2] Foley, B.J. "Designing Visualization Tools for Learning." CHI '98: CHI 98 Conference Summary on Human Factors in Computing Systems, Los Angeles, Calif., 1998, pp.309-310.

[3] Gramoll, K. "Using 'Working Model' to Introduce Design into a Freshman Engineering Course." 1994 ASEE Conf Proc., Edmonton, Canada, June 1994.

[4] John D. McGervey, "Student Measurement of Fermi Energy by Positron Annihilation”, 1963.

[5] Keithley Application notes by www.keithley.com

[6] G.S.Georgiev, G.T.Georgiev, S.L.Stefanova, virtual instruments - functional model, organization and programming architecture, International Journal "Information Theories \& Applications" Vol.10. 
[7] Product manuals from www.ni.com.

[8] S. M. Sze, Physics of Semiconductor Devices, 2nd ed. Wiley Interscience, New York, 1981.

[9] Blood P, Orton J W. The electrical characterization of semiconductors: majority carriers and electron states. AcademicPress, 1992.

[10] Orton J W, Blood P. The electrical characterization of semiconductors: measurement of minority carrier properties. Academic Press, 1990.

[11] Minami T. Transparent conducting oxide semiconductors for transparent electrodes. Semiconductor Science Technology, 2005, 20: S35.

[12] $\mathrm{Wu} \mathrm{X}$. High-efficiency polycrystalline thin-film solar cells. Solar Energy, 2004, 77: 803.

[13] Gessert T A, Metzger W K, Dippo P, et al. Dependence of carrier lifetime on copper-contacting temperature and $\mathrm{ZnTe}: \mathrm{Cu}$ thickness in $\mathrm{CdS} / \mathrm{CdTe}$ thin film solar cells. Thin Solid Films, 2009, 517: 2370.

[14] Look D C. Semi-conductors and semi-metals. Willardson R K, Beer A C, ed. New York: Academic Press, 1983.

\section{AUTHORS PROFILE}

Dr. K V Gangadharan is currently a Professor in Mechanical Engineering Department at National Institute of Technology Karnataka, India. He had received a Ph.D. from
IIT Madras, India and a M.Tech (NIT Trichy), B. Tech (Calicut University).He is working on Vibration and Control, Dynamics, FEM, Condition Monitoring, Experimental Methods in Vibration .He is Professor Since 1993 at NITK Surathkal .His research interests are vibration and vibration control, smart materials and its applications, dynamic properties of materials - damping study and experimental method in dynamics and vibration related environmental impact assessment.Dr. K V Gangadharan has thirty five (35) international and national conferences paper and Eight (8) journals paper. He established Solve Lab at NITK Surathkal under Centre for system design.

Prashant Kumar Tripathi is currently pursuing M.Tech Mechatronics by Research in Mechanical Engineering Department at National Institute of Technology Karnataka. He obtained Bachelor of Engineering degree in Mechanical Engineering from S.J.C Institute of Technology in the year 2009.His area of research is computer application development for Remote Labs and Robotics. He has Six (6) international and national conferences papers and one (1) international journal paper. $\mathrm{He}$ is also interested in the use of visualization tools in various applications. In addition to educational interests, $\mathrm{He}$ is an active researcher in the field of Visualization of vibration and its control in mechanical system (2011). 